the pooled single-cell gene-expression data sets from human and mouse cerebral cortex and again used a clustering analysis to identify matching cell types. Using this method, the authors determined that almost all of the 75 cell types identified in the human cortex showed correspondence (homology) with cell types described in the mouse. They also found, however, that the diversity of neurons in cortical layers I-IV, which are thought to be responsible for many aspects of cognition specific to primates ${ }^{10}$, is greater in human cortex than in mouse cortex. Moreover, the authors noted that, within homologous cell types, the expression of certain genes was highly variable between species, including genes used to define these cell types in either species' data set (Fig. 1b). Microglia, the immune cells of the brain, showed the greatest divergence in gene expression between humans and mice, suggesting that the role of microglia in neuroimmune disorders might differ considerably between species.

One shortcoming of Hodge and colleagues' study is the use of data that were obtained by different profiling methods (the authors profiled single nuclei from the human samples, whereas whole cells were profiled in the mouse analysis) and from different cortical areas (temporal lobe in the human and visual cortex in the mouse). Nevertheless, the analysis demonstrates a new approach that could one day enable a thorough comparison of cell-type homology across many species ${ }^{11}$. Notably, genes that encode certain receptors for neurotransmitter molecules were among the genes that showed the greatest divergence in expression between human and mouse, suggesting that the authors' observations could have implications for attempts to model the function of synaptic connections between neurons, and their disorders.

Hodge and colleagues' study advances our understanding of the cellular composition of the cerebral cortex, and reveals previously unrecognized distinctions between the organization of this brain region in humans and mice. The authors' comparison of conserved cell types in humans and mice calls for further investigation of how cell types have been conserved during evolution and the importance of species-specific changes to the recipe for the brain.

Matthew G. Keefe and Tomasz J. Nowakowski are in the Departments of Anatomy and of Psychiatry, University of California, San Francisco, California 94143, USA. T.J.N. is also at the Chan Zuckerberg Biohub, San Francisco.

e-mail: tomasz.nowakowski@ucsf.edu

1. Hodge, R. D. et al. Nature 573, 61-68 (2019)

2. Shepherd, G. M. et al. Front. Neuroanat. 13, 25 (2019).

3. Hawrylycz, M. J. et al. Nature 489, 391-399 (2012).

4. Oldham, M. C. et al. Nature Neurosci. 11, 1271-1282 (2008)

5. Darmanis, S. et al. Proc. Natl Acad. Sci. USA 112, 7285-7290 (2015).

6. Zeng, H. et al. Cell 149, 483-496 (2012).

7. Rakic, P. Science 183, 425-427 (1974).

8. Tasic, B. et al. Nature 563, 72-78 (2018).

9. Nowakowski, T. J. et al. Science 358, 1318-1323 (2017).

10.Geschwind, D. H. \& Rakic, P. Neuron 80, 633-647 (2013).

11.Tosches, M. A. \& Laurent, G. Curr. Opin. Neurobiol. 56, 199-208 (2019).

This article was published online on 21 August 2019.
Fluorine and amide groups together at last

A practical method has been devised to synthesize compounds that have previously been almost impossible to make. The combination of fluorine atoms and amide groups in the compounds might be useful in pharmaceuticals. SEE LETTER P.102

\section{JONATHAN CLAYDEN}

$\mathrm{I}$ $\mathrm{n}$ medicinal chemistry, the chemical group known as the amide is king. Amides consist of a nitrogen atom linked to a carbonyl group $(\mathrm{C}=\mathrm{O})$, and featured in all but four of the top 40 bestselling drugs in 2018 (see go.nature. com/30f709w). On page 102, Scattolin et al. ${ }^{1}$ expand the range of amides available for drug discovery by showing how to synthesize a previously inaccessible group of compounds known as $\mathrm{N}$-trifluoromethylamides, in which the amide is closely associated with three fluorine atoms.

Amides are prevalent in medicinally important compounds not only because they are particularly stable, but also because they are polar (they contain regions of high positive and negative electrical charge density), which allows amide-containing drugs to interact with biological receptors and enzymes. In these respects, the use of amides in drugs follows nature's example - amide groups provide the links between amino-acid residues in proteins. But drugs don't just have to interact with biological targets; they must also resist rapid metabolic degradation in the complex environment of the human body.

One well-established way to protect molecules from such degradation involves the use of fluorine atoms. Like amides, carbon-fluorine $(\mathrm{C}-\mathrm{F})$ bonds are polar and unreactive. But, unlike amides, they are almost entirely alien to biology - which means that metabolic enzymes struggle to degrade them rapidly. This makes the incorporation of fluorine atoms an effective way to increase the metabolic stability, as well as other desirable properties, of drug compounds ${ }^{2}$. atoms can be a particularly fruitful strategy for drug development - indeed, the bestselling drug of all time, cholesterol-reducing atorvastatin, contains both an amide and a fluorine substituent, albeit in different parts of the molecule. But the synthesis of molecules in which amides and fluorine atoms are closely associated is far from straightforward. Attempts to assemble fluorinated amides typically require reaction conditions that can cause the degradation of sensitive chemical groups in the target molecule.

Scattolin et al. describe a practical solution for the synthesis of an otherwise almost inaccessible family of fluorinated amides that contain an $\mathrm{N}$-trifluoromethyl group
Combining amide groups with fluorine

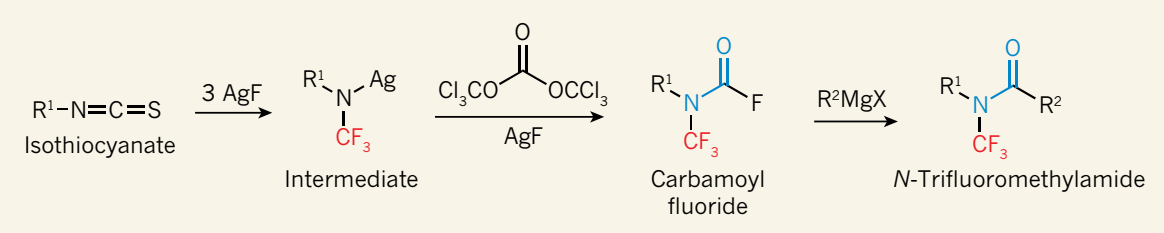

Figure 1 | The synthesis of $N$-trifluoromethylamide compounds. $N$-trifluoromethylamides contain a trifluoromethyl group (red) attached to an amide group (blue), and have been almost impossible to make. $\mathrm{R}^{1}$ and $\mathrm{R}^{2}$ represent any chemical group. Scattolin et al. ${ }^{1}$ report a practical synthesis of these compounds, which are of interest for drug discovery. The authors treated an isothiocyanate with silver fluoride (AgF), producing an intermediate compound in which a trifluoromethyl group is attached to a nitrogen atom. This intermediate reacts with bis(trichloromethyl) carbonate $\left(\mathrm{CO}\left(\mathrm{OCCl}_{3}\right)_{2}\right)$ in the presence of silver fluoride to make a carbamoyl fluoride. Treating this compound with magnesium-containing reagents known as Grignard reagents ( $\mathrm{R}^{2} \mathrm{MgX}$, where $\mathrm{X}$ is a halogen atom) produces $\mathrm{N}$-trifluoromethylamides. Side products of the reactions are not shown. 
$\left(\mathrm{N}-\mathrm{CF}_{3} ;\right.$ Fig. 1). The authors cleverly overcome the reluctance of fluoride ions to take part in useful reactions by using a relatively reactive salt, silver fluoride, to promote bond formation between two reagents that will be familiar to organic chemists but are rarely combined. The first of these is a sulfur-containing compound called an isothiocyanate (Fig. 1) - more specifically, the compound that promotes the classical Edman degradation reaction, used in early methods for determining the amino-acid sequence of proteins. Researchers from the same group as Scattolin et al. previously used ${ }^{3,4}$ silver ions to promote the replacement of sulfur atoms by fluorine. Silver fluoride has the same role in the present work: it rips out sulfur from the Edman reagent and replaces it with three fluorine atoms, forming an intermediate compound that contains a trifluoromethyl $\left(\mathrm{CF}_{3}\right)$ group.

The second reagent is bis(trichloromethyl) carbonate (Fig. 1), which is often used to make amides and amide-like derivatives ${ }^{5}$. The authors report that, when used in combination with silver fluoride, this carbonate traps the typically highly unreactive $\mathrm{CF}_{3}$-containing intermediate to form a compound called a carbamoyl fluoride. It is this compound that forms the conceptual breakthrough of Scattolin and colleagues' work.

The carbamoyl fluoride already contains the carbon-nitrogen bond of the target amide, thereby sidestepping the usual difficulties associated with making fluorinated amides. Moreover, it is stable enough to be isolated, but reactive enough to act as a building block for the synthesis of a range of $\mathrm{N}$-trifluoromethylamides - which Scattolin et al. prepare by reacting the carbamoyl fluoride with various magnesium-containing compounds known as Grignard reagents (Fig. 1). The authors show that chemical groups called ureas and carbamates can also be made in this final step, widening the applicability of the chemistry beyond fluorinated amides.

In this work, Scattolin and colleagues have impressively choreographed the reactions of several seemingly incompatible, highly reactive reagents, perfectly controlling which meets which, and when. The authors demonstrate that their method can be used to make not only simple $\mathrm{N}$-trifluoromethylamide molecules, but also chemically sensitive ones such as those based on amino acids, drug scaffolds and the monomers used to make polymeric materials.

The reported reactions will enable medicinal chemists to prepare previously unavailable compounds for testing in drug-discovery programmes - some of these compounds might well display new biological activities. The addition of a methyl group to the nitrogen atom of an amide in drug candidates has long been used to alter the conformations adopted by those molecules ${ }^{6}$, thereby altering their biological activities; the ability to make $N$-trifluoromethylamides will broaden the scope of that strategy.
There are, however, drawbacks to the new chemistry that will need to be addressed before it can be used on the industrial scales needed to manufacture a drug. The main issue is that the reactions require substantial quantities of the necessary reagents: every fluorine atom introduced into a molecule, plus two more that are expelled during the reaction, is accompanied by an atom of silver, meaning that five atoms of silver are needed for every molecule of product. This is acceptable for the initial stages of drug discovery (lead development and optimization), which typically involve only milligram to gram quantities of drug candidates. However, it would not be sustainable for synthesizing compounds on the kilogram scales needed for testing in clinical trials, let alone for industrial manufacturing processes, because of the cost and large amount of waste produced. Another breakthrough will be needed to find a way of synthesizing $N$-trifluoromethylamides sustainably on such large scales.

Looking beyond medicinal chemistry,
Scattolin and co-workers' findings reveal that isothiocyanate groups can act as precursors of trifluoromethyl groups bonded to nitrogen atoms. Their reactions might find much wider use in chemical synthesis, where the ability to make trifluoromethyl-substituted nitrogen compounds could facilitate the design and synthesis of catalysts or materials with new properties.

Jonathan Clayden is at the School of Chemistry, University of Bristol, Bristol BS8 1TS, UK.

e-mail:j.clayden@bristol.ac.uk

1. Scattolin, T., Bouayad-Gervais, S. \& Schoenebeck, F. Nature 573, 102-107 (2019).

2. Purser, S., Moore, P. R., Swallow, S. \& Gouverneur, V. Chem. Soc. Rev. 37, 320-330 (2008)

3. Tyrra, W. J. Fluor. Chem. 109, 189-194 (2001).

4. Scattolin, T., Deckers, K. \& Schoenebeck, F. Angew. Chem. Int. Edn 56, 221-224 (2017).

5. Eckert, H. \& Forster, B. Angew. Chem. Int. Edn 26 894-895 (1987)

6. Yamaguchi, K. et al. J. Am. Chem. Soc. 113, 5474-5475 (1991)

\section{A lid for the marker}

\section{Two reports examine how a protein complex that adds transcription-repressing marks to histone proteins is potently inhibited by the protein EZHIP during the maturation of sperm and egg cells and in a type of brain cancer.}

\section{SERGI ARANDA \& LUCIANO DI CROCE}

$\mathrm{H}$ ow can a single genome generate specialized cells at the correct time and place in multicellular organisms? Gene-expression programs that are specific to different cell types are regulated by the addition and removal of chemical groups, known as epigenetic marks, to and from chromatin the compact protein-DNA complex in which the genome is organized. Two papers in Nature Communications (one by Ragazzini et al. ${ }^{1}$ and the other by Jain et al. ${ }^{2}$ ), together with recent studies ${ }^{3-5}$, show how a protein called EZHIP can regulate and disrupt the addition of a major epigenetic mark by the Polycomb repressive complex 2 (PRC2) during certain biological events and in a type of cancer.

The Polycomb protein complexes are key epigenetic modulators that act to repress gene expression ${ }^{6}$. Mammalian PRC2 contains a core complex consisting of several subunits, including either EZH1 or EZH2 as an enzyme subunit ${ }^{7}$. The core complex can interact with various accessory subunits that influence its enzymatic activity and direct it to specific regions of the genome.

The major substrate of PRC2 is a nucleosome: a complex of eight histone proteins, around which DNA is wrapped; nucleosomes are the basic building blocks of chromatin.
PRC2 adds a trimethyl group (me3) to a specific amino-acid residue of the $\mathrm{H} 3$ histone (lysine 27; abbreviated as K27), generating an epigenetic mark termed H3K27me3 (Fig. 1a). This modification occurs initially at certain sites and promotes the passage of PRC2 across adjacent regions of the chromatin ${ }^{8}$.

The distribution of the H3K27me3 mark on nucleosomes throughout the genome changes drastically during certain biological events, including in sperm and egg cells when they mature, and in fertilized egg cells ${ }^{9}$. However, the molecular mechanisms that regulate this redistribution, and its functional consequences, are unknown. EZHIP is expressed only in the testes, ovaries and embryo-surrounding tissues of placental mammals ${ }^{10}$, and was previously shown to interact with PRC2 in cultured mouse stem cells ${ }^{11,12}$. Ragazzini and colleagues demonstrate that, in the testes and ovaries of mice, EZHIP binds to and reduces the enzymatic activity of PRC2.

The authors also show that mice lacking EZHIP can live and develop normally, and are apparently fertile, but that female mice that lack EZHIP show progressive infertility with age. This finding calls for a re-evaluation of genomic studies in women with infertility, to clarify whether certain EZHIP gene variants could increase the risk of this condition. EZHIP protein is found 